
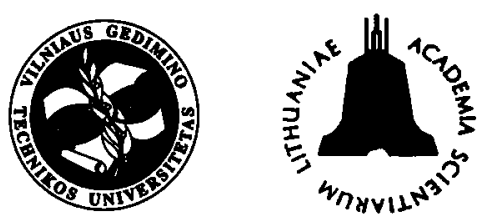

\author{
JOURNAL OF CIVIL ENGINEERING AND MANAGEMENT \\ http:/www.vtu.lt/english/editions
}

2003, Vol $L X$, No 3, 218-224

\title{
ON THE INTERACTION OF ELASTIC WAVES
}

\author{
Aleksandras Krylovas ${ }^{1}$, Raimondas Čiegis ${ }^{2}$ \\ Dept of Mathematical Modelling, Vilnius Gediminas Technical University, \\ Sauletekio al. 11, LT-2040 Vilnius, Lithuania \\ 1 e-mail: akr@fm.vtu.lt ; 2 e-mail:rc@fm.vtu.lt \\ Received 07 Oct 2002; accepted 12 Febr 2003
}

\begin{abstract}
The non-linear mathematical model of the interaction of elastic waves is presented. The conditions of possible resonant interaction of periodic waves are described. The method of internal averaging for getting uniformly valid asymptotic expansions is used in both, ie resonant and non-resonant, cases. Results of numerical experiments are presented for the resonant interaction of the elastic waves.

Keywords: elastic waves interaction, non-linear waves, perturbation method, asymptotic analysis, averaging equations.
\end{abstract}

\section{Introduction}

The wave equation

$$
u_{t t}-c^{2} u_{x x}=0
$$

models propagation of different types of waves such as elastic waves in an elastic string, membrane, or solid, sound waves in a gas, electromagnetic waves. The simple linear equation is derived using the assumption that the perturbations $|u|$ are small. It is important that a solution of mathematical model (1) should not necessary be small. It is well known that the solution of (1) equation is given in the form

$$
u=\varphi(x-c t)+\psi(x+c t),
$$

where $\varphi(x-c t)$ corresponds to a wave propagation with speed $c$ in the positive direction and $\psi(x+c t)$ corresponds to a wave propagation in the negative direction. Hence any solution of (1) cannot be used to model the real wave propagation process [1].

The second restriction of the mathematical model (1), which is also frequently not taken into account, deals with the fact that equation (1) describes real world processes only for a finite time interval. We again note that the linear combination $\varphi+\psi$ describes a solution of (1) for all $0<t<+\infty$.

In this paper we derive a more accurate mathematical model for describing the wave propagation in elastic materials and propose a new method for constructing the asymptotic approximation of the solution.

Perturbation methods are widely used for the analysis of wave phenomena $[2,3]$. Application of these methods in the elastic waves theory has many special features, thus many works are devoted to this subject [4-6]. Our algorithm is similar to the well-known ray method [7, 8]. For a list of applications of (1) see [9, $10]$. We note that the method of solving the elastic wave interaction problem proposed in [9] cannot be used in the case of resonant interaction of periodic waves. The main idea of our algorithm is based on a special type of averaging operator. Averaging methods are widely used in asymptotic analysis (e g [11-13]). The new feature of our method is based on application of averaging operator for functions, which are solutions of obtained averaged equations. Thus we get integro-differential problems. Such a modification of the algorithm gives us a possibility to describe the resonant interactions which can arise in such systems. Mathematical modelling of interaction of elastic waves is important for some industrial applications [14, 15].

The rest of the paper is organised as follows. In Section 2 we formulate a non-linear one-dimensional mathematical model of propagation of elastic waves. The analysis of some simplified models is presented in Section 3 . We are mainly concerned to give a sampling of specific problems that are important in the asymptotic analysis of differential equations involving a small parameter $\varepsilon$. In Section 4 we present a method of averaging and construct asymptotic approximation, which is uniformly valid for $t=O\left(\frac{1}{\varepsilon}\right)$. Using this method we obtain the averaged system of integro-differential equations. Conditions describing a possibility of resonant interaction of waves for periodic solutions are presented. The results of numeric experiments are given in Section 5. 


\section{Mathematical model}

Let's consider the problem of wave propagation in two-dimensional elastic materials. We assume that displacements do not depend on the $y$ coordinate and therefore the mathematical model will be one-dimensional. Restricting our attention to the axial displacements along $x$ and $y$ directions, we have equations [16]:

$$
\left\{\begin{array}{l}
\rho u_{t t}=\sigma_{x}, \\
\rho v_{t t}=\tau_{x} .
\end{array}\right.
$$

Here $\rho$ is the density of the material, $\sigma$ and $\tau$ are longitudinal normal and shear stresses along the $x$-axis. The linear wave equation (1) follows from (2) if we use assumptions

$$
\sigma=(\lambda+2 \mu) u_{x}, \quad \tau=\mu v_{x},
$$

here $\lambda$ and $\mu$ are the Lamé coefficients. The equations (3) are obtained by using the simple approximation for the full energy of the system

$$
F \approx F_{0}+F_{11} u_{x}^{2}+F_{22} v_{x}^{2}
$$

then $\sigma=\frac{\partial F}{\partial u_{x}}$ and $\tau=\begin{aligned} & \partial F \\ & \partial v_{x}\end{aligned}[16]$.

In order to get high-order approximations we use more terms in a Taylor series of $F$ :

$$
\left\{\begin{array}{l}
\sigma=(\lambda+2 \mu) u_{x}+4 \rho\left(a_{1} u_{x}^{2}+a_{2} u_{x} v_{x}+a_{3} v_{x}^{2}\right) \\
\tau=\mu v_{x}+4 \rho\left(b_{1} u_{x}^{2}+b_{2} u_{x} v_{x}+b_{3} v_{x}^{2}\right)
\end{array}\right.
$$

Let's introduce a dimensionless small parameter $\varepsilon$. We seek a solution in the form

$$
\begin{aligned}
& u(y, x)=u_{0}+\varepsilon u_{1}(t, x) \\
& v(y, x)=v_{0}+\varepsilon v_{1}(t, x)
\end{aligned}
$$

Substituting (5) into the system of equations (2), (4) and separating terms according to their associated power of $\varepsilon$, we derive the following problem:

$$
\left\{\begin{array}{l}
\varepsilon \rho u_{1 t t}=\varepsilon(\lambda+2 \mu) u_{1 x x} \\
+4 \varepsilon^{2} \rho \frac{\partial}{\partial x}\left(a_{1} u_{1 x}^{2}+a_{2} u_{1 x} v_{1 x}+a_{3} v_{1 x}^{2}\right) \\
\varepsilon \rho v_{1 t t}=\varepsilon \mu v_{1 x x} \\
+4 \varepsilon^{2} \rho \frac{\partial}{\partial x}\left(b_{1} u_{1 x}^{2}+b_{2} u_{1 x} v_{1 x}+b_{3} v_{1 x}^{2}\right)
\end{array}\right.
$$

We note that all constants $u_{0}$ and $v_{0}$ satisfy the system (2), (4) and equations (6) do not depend on these constants. Therefore we can take $u_{0}=v_{0}=0$ in (5).

Let's denote derivatives

$$
\begin{aligned}
& u_{1 x}=P(t, x), v_{1 x}=Q(t, x), \\
& u_{1 t}=R(t, x), v_{1 t}=S(t, x) .
\end{aligned}
$$

Then we get that

$$
u_{1 t t}=R_{t}, u_{1 x x}=P_{x}, v_{1 t t}=S_{t}, v_{1 x x}=Q_{x}
$$
and rewrite the system (5) in the following form:

$$
\left\{\begin{array}{l}
R_{t}-\frac{\lambda+2 \mu}{\rho} P_{x}=4 \varepsilon \frac{\partial}{\partial x}\left(a_{1} P^{2}+a_{2} P Q+a_{3} Q^{2}\right), \\
S_{t}-\frac{\mu}{\rho} Q_{x}=4 \varepsilon \frac{\partial}{\partial x}\left(b_{1} P^{2}+b_{2} P Q+b_{3} Q^{2}\right) .
\end{array}\right.
$$

Using the well-known Riemann invariants, we can derive a symmetric form of this system. To do this, we denote axial and vertical velocities of waves by

$$
c_{p}=\sqrt{\frac{\lambda+2 \mu}{\rho}}, c_{s}=\sqrt{\frac{\mu}{\rho}}
$$

and introduce four unknown functions

$$
p^{ \pm}=R \pm c_{p} P, s^{ \pm}=S \pm c_{s} Q .
$$

Then we have that

$$
\begin{aligned}
& R=\frac{1}{2}\left(p^{+}+p^{-}\right), P=\frac{1}{2 c_{p}}\left(p^{+}-p^{-}\right), \\
& S=\frac{1}{2}\left(s^{+}+s^{-}\right), Q=\frac{1}{2 c_{s}}\left(s^{+}-s^{-}\right) .
\end{aligned}
$$

Using equalities $P_{t}=R_{x}, Q_{t}=S_{x}$, we get from (8) the following system of four equations:

$$
\left\{\begin{array}{l}
p_{t}^{ \pm} \mp c_{p} p_{x}^{ \pm}=\varepsilon_{\partial x}^{\partial} a\left(p^{+}, p^{-}, s^{+}, s^{-}\right), \\
s_{t}^{ \pm} \mp c_{s} s_{x}^{ \pm}=\varepsilon{ }_{\partial x}^{\partial} b\left(p^{+}, p^{-}, s^{+}, s^{-}\right), \\
a=a_{1}\left(\begin{array}{c}
p^{+}-p^{-} \\
c_{p}
\end{array}\right)^{2}+a_{2} s^{+}-s^{-}, p^{+}-p^{-} \\
c_{p} \\
+a_{3}\left(\begin{array}{c}
s^{+}-s^{-} \\
c_{s}
\end{array}\right)^{2}, \\
b=b_{1}\left(\begin{array}{c}
p^{+}-p^{-} \\
c_{p}
\end{array}\right)^{2}+b_{2} s^{+}-s^{-} . p^{+}-p^{-} \\
c_{s} \\
+b_{3}\left(\begin{array}{c}
s^{+}-s^{-} \\
c_{s}
\end{array}\right)^{2}
\end{array}\right.
$$

Initial and boundary conditions must be also specified in order to define a unique solution. This conditions will be given later.

\section{Asymptotic analysis}

Our aim is to investigate the dependence of the solution of system (12) on a small parameter $\varepsilon$ and to illustrate the failure of the regular expansion. We note 
that for $\varepsilon=0$ the system is reduced to four independent equations and the solution can be easily calculated in the form

$$
\begin{aligned}
& p^{+}=\varphi_{1}\left(x+c_{p} t\right), p^{-}=\varphi_{2}\left(x-c_{p} t\right), \\
& s^{+}=\psi_{1}\left(x+c_{s} t\right), s^{-}=\psi_{2}\left(x-c_{s} t\right) .
\end{aligned}
$$

Thus we have

$$
\left\{\begin{aligned}
u_{1 t} \equiv R & =\frac{1}{2}\left(\varphi_{1}+\varphi_{2}\right) \\
v_{1 t} \equiv S & =\frac{1}{2}\left(\Psi_{1}+\Psi_{2}\right) \\
u_{1}(t, x) & =\frac{1}{2}\left(\int \varphi_{1}\left(x+c_{p} t\right) d t+\int \varphi_{2}\left(x-c_{p} t\right) d t\right) \\
& =\frac{1}{2 c_{p}}\left(\Phi_{1}\left(x+c_{p} t\right)-\Phi_{2}\left(x-c_{p} t\right)\right) \\
v_{1}(t, x) & =\frac{1}{2}\left(\int \Psi_{1}\left(x+c_{s} t\right) d t+\int \Psi_{2}\left(x-c_{s} t\right) d t\right) \\
& =\frac{1}{2 c_{s}}\left(\Psi_{1}\left(x+c_{s} t\right)-\Psi_{2}\left(x-c_{s} t\right)\right)
\end{aligned}\right.
$$

Therefore for $\varepsilon=0$ system (12) is equivalent to system (2), (3). In order to motivate our discussion of problem (12) with a small parameter and to show the role of special asymptotic expansion, let us first study the scalar problem

$$
\left\{\begin{array}{l}
p_{t}-c_{p} p_{x}=\varepsilon p p_{x}, \\
p(0, x ; \varepsilon)=0
\end{array}\right.
$$

Simple calculations enable us to express the solution of (13) in the form of the implicit equation

$$
p=\sin \left(x+c_{p} t+\varepsilon t p\right)
$$

Therefore the derivatives of the solution are given by

$$
\begin{aligned}
& p_{t}=\frac{\left(c_{p}+\varepsilon p\right) \cos \left(x+c_{p} t+\varepsilon t p\right)}{1-\varepsilon t \cos \left(x+c_{p} t+\varepsilon t p\right)}, \\
& p_{x}=\frac{\cos \left(x+c_{p} t+\varepsilon t p\right)}{1-\varepsilon t \cos \left(x+c_{p} t+\varepsilon t p\right)} .
\end{aligned}
$$

Let us assume that $x+c_{p} t=0$. Then we obtain from (14) that

$$
p=0, \cos \left(x+c_{p} t+\varepsilon t p\right)=1 .
$$

Thus denominators of $p_{t}$ and $p_{x}$ are equal to zero when $t=\frac{1}{\varepsilon}$. A purely non-linear effect of wave sharpening is obtained. Fig 1 presents solutions at two different time moments, where we denote $y=x+c_{p} t, T=\varepsilon t$.

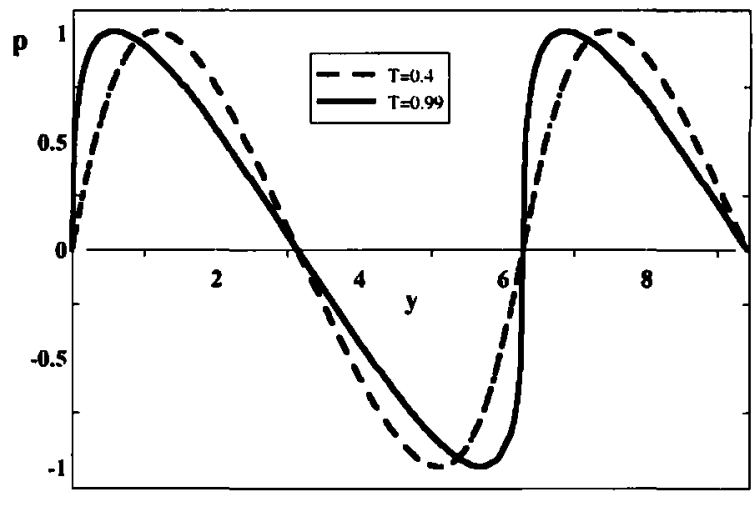

Fig 1. The graph of non-linear wave (14)

Let us consider the Taylor expansion of the function

$$
\begin{aligned}
& p(t, x ; \varepsilon)=\sin \left(x+c_{p} t\right) \\
& +\varepsilon t \sin \left(x+c_{p} t\right) \cos \left(x+c_{p} t\right)+O\left((\varepsilon t)^{2}\right) .
\end{aligned}
$$

Thus the wave approximation

$$
p(t, x ; \varepsilon) \approx \sin \left(x+c_{p} t\right)
$$

is uniformly valid only while $\varepsilon t$ is a small number. In this case we use the linear-wave equation. In order to compute the solution for $\varepsilon t=1$ we must consider the complete non-linear problem (13). Now the expansion (16) is not useful as an approximation of the solution and special expansion procedures must be applied in order to get uniformly converging approximations.

An interesting feature of (16) is that it contains a second mode of the Fourier expansion

$$
\sin \left(x+c_{p} t\right) \cos \left(x+c_{p} t\right)=\frac{1}{2} \sin 2\left(x+c_{p} t\right),
$$

though the initial condition describes only the first mode. This is a direct consequence of non-linearity of the differential equation. We will get high order modes in (16) if more terms are taken into account. In general, the influence of these modes is small but in some cases the effect of resonant interaction is observed. To illustrate such a possibility let us consider the first equation of system (12)

$$
p_{t}^{+}-c_{p} p_{x}^{+}=\varepsilon s^{-} s_{x}^{+},
$$

and assume that functions $s^{-}$and $s^{+}$are known a priori: $s^{-}=\sin k\left(x-c_{s} t\right), s^{+}=\sin l\left(x+c_{s} t\right), k, l \in \mathrm{R}$.

We take the initial condition

$$
p^{+}(0, x ; \varepsilon)=0 .
$$

Denoting the right-hand side of equation (17) by $\varepsilon f(t, x)=$ $\frac{\varepsilon l}{2}\left(\sin \left((k+l) x+(k-l) c_{s} t\right)+\sin \left((k-l) x+(k+l) c_{s} t\right)\right)$ 
and integrating (17) along the characteristic, we obtain

$$
p^{+}(t, x ; \varepsilon)=\varepsilon \int_{0}^{t} f\left(r, x+c_{p} t-c_{p} r\right) d r .
$$

If for all $k, l \in Z$ the following conditions are satisfied

$$
\left\{\begin{array}{l}
(k+l) c_{p} \neq(k-l) c_{s} \\
(k-l) c_{p} \neq(k+l) c_{s}
\end{array}\right.
$$

then the integral on right-hand side of equation (19) is equal to a sum of trigonometric functions and $p^{+}$decreases uniformly for all $t, x \in R$ when $\varepsilon \rightarrow 0$. The situation changes if at least one of conditions (20) is not satisfied, then a resonant factor $t$ will arise in (19).

Let us consider the problem (17), (18) with

$$
c_{p}=1, c_{s}=2, k=3, l=1 .
$$

Then we obtain the solution in the explicit form

$$
p^{+}=\varepsilon t \sin 4(x+t)+\frac{1}{6} \cos 2(x+t)-\frac{1}{6} \cos (8 t+2 x)
$$

and the first term on the right-hand side of this equality defines the secular term.

\section{The method of internal averaging}

It follows from the previous analysis that we cannot express a solution of (12) by a simple Taylor expansion

$$
\begin{aligned}
& p^{ \pm}(t, x ; \varepsilon)=p_{0}^{ \pm}(t, x)+\varepsilon p_{1}^{ \pm}(t, x)+\cdots, \\
& s^{ \pm}(t, x ; \varepsilon)=s_{0}^{ \pm}(t, x)+\varepsilon s_{1}^{ \pm}(t, x)+\cdots
\end{aligned}
$$

Application of such a method for solving problem (13),(14) gives expansion (16), and this example was investigated above. We have proved that approximation

(16) cannot be used for $t \approx \frac{1}{\varepsilon}$.

The system (12) is a particular case of general hyperbolic systems of differential equations, which are investigated in our papers $[17,18]$ (see also references to related works). In this article we will present main mathematical results and will omit the proofs.

We apply the multiple-scale analysis to (12) and introduce a slow time $\tau=\varepsilon t$ and four fast characteristic dependent variables

$$
y^{ \pm}=x \pm c_{p} t, z^{ \pm}=x \pm c_{s} t .
$$

We also define average values of the function $f$, which are computed along characteristics

$$
\begin{aligned}
& M_{y^{+}}[f]= \\
& \lim _{T \rightarrow \infty} \frac{1}{T} \int_{0}^{T} f\left(\tau, y^{+}, y^{+}-2 c_{p} r, y^{+}+\left(c_{s}-c_{p}\right) r, y^{+}-\left(c_{s}+c_{p}\right) r\right) d r
\end{aligned}
$$

$$
\begin{aligned}
& M_{y^{-}}[f]= \\
& \lim _{T \rightarrow \infty} \frac{1}{T} \int_{0}^{T} f\left(\tau, y^{-}+2 c_{p} r, y^{-}, y^{-}+\left(c_{s}+c_{p}\right) r, y^{-}+\left(c_{p}-c_{s}\right) r\right) d r
\end{aligned}
$$$$
M_{z}+[f]=
$$$$
\lim _{T \rightarrow \infty} \frac{1}{T} \int_{0}^{T} f\left(\tau, z^{+}-\left(c_{s}-c_{p}\right) r, z^{+}-\left(c_{s}+c_{p}\right) r, z^{+}, z^{+}-2 c_{s} r\right) d r
$$

$M_{z^{-}}[f]=$

$\lim _{T \rightarrow \infty} \frac{1}{T} \int_{0}^{T} f\left(\tau, z^{-}+\left(c_{s}+c_{p}\right) r, z^{-}+\left(c_{s}-c_{p}\right), z^{-}+2 c_{s} r, z^{-}\right) d r$.

Each integral depends only on one characteristic dependent variable. We look for asymptotic approximations given in the form

$$
p^{ \pm}(t, x ; \varepsilon)=P^{ \pm}\left(\tau, y^{ \pm}\right), s^{ \pm}(t, x, \varepsilon)=S^{ \pm}\left(\tau, z^{ \pm}\right),
$$

where $P^{ \pm}$and $S^{ \pm}$satisfy the averaged integro-differential system

$$
\left\{\begin{array}{l}
P_{\tau}^{ \pm}-\frac{2 a_{1}}{c_{p}^{2}} P^{ \pm} P_{y^{ \pm}}^{ \pm}= \\
-\frac{2 a_{3}}{c_{s}^{2}} M_{y^{ \pm}}\left[\frac{\partial}{\partial x}\left(S^{+} S^{-}\right)\right] \mp \\
\frac{a_{2}}{c_{s} c_{p}} M_{y^{ \pm}}\left[\frac{\partial}{\partial x}\left(P^{\mp}\left(S^{+}-S^{-}\right)\right)\right] \\
S_{\tau}^{ \pm}-\frac{2 b_{3}}{c_{s}^{2}} S^{ \pm} S_{z^{ \pm}}^{ \pm}= \\
-\frac{2 b_{1}}{c_{p}^{2}} M_{z^{ \pm}}\left[\frac{\partial}{\partial x}\left(P^{+} P^{-}\right)\right] \mp \\
\frac{b_{2}}{c_{s} c_{p}} M_{z^{ \pm}}\left[\frac{\partial}{\partial x}\left(S^{ \pm}\left(P^{+}-P^{-}\right)\right)\right]
\end{array}\right.
$$

Here we use notation:

$$
\begin{aligned}
& \frac{\partial}{\partial x}\left(S^{+} S^{-}\right)=S_{z^{+}}^{+} S^{-}+S^{+} S_{z^{-}}^{-}, \\
& \partial\left(P^{+} P^{-}\right)=P_{y^{+}}^{+} P^{-}+P^{+} P_{y^{-}}^{-}
\end{aligned}
$$

$$
\begin{aligned}
& \frac{\partial}{\partial x}\left(P^{\mp}\left(S^{+}-S^{-}\right)\right)=P_{y^{\mp}}^{\mp}\left(S^{+}-S^{-}\right)+P^{\mp}\left(S_{z^{+}}^{+}-S_{z^{-}}^{-}\right), \\
& \frac{\partial}{\partial x}\left(S^{\mp}\left(P^{+}-P^{-}\right)\right)=S_{z^{\mp}}^{\mp}\left(P^{+}-P^{-}\right)+S^{\mp}\left(P_{y^{+}}^{+}-P_{y^{-}}^{-}\right) .
\end{aligned}
$$


Let us assume that displacements $u$ and $v$ satisfy the following initial conditions

$$
\begin{aligned}
& u(0, x)=\varepsilon \varphi_{0}(x), u_{t}(0, x)=\varepsilon \varphi_{1}(x), \\
& v(0, x)=\varepsilon \psi_{0}(x), v_{t}(0, x)=\varepsilon \psi_{1}(x) .
\end{aligned}
$$

Substituting (24) into (7) leads to the following initial conditions for $P, Q, R$ and $S$ :

$$
\begin{aligned}
& P(0, x)=\varphi_{0}^{\prime}(x), Q(0, x)=\psi_{0}^{\prime}(x), \\
& R(0, x)=\varphi_{1}(x), S(0, x)=\psi_{1}(x) .
\end{aligned}
$$

Let us define new functions

$$
\begin{aligned}
& p_{0}^{ \pm}(x)=\varphi_{1}(x) \pm c_{p} \varphi_{0}^{\prime}(x), \\
& s_{0}^{ \pm}(x)=\psi_{1}(x) \pm c_{s} \psi_{0}^{\prime}(x) .
\end{aligned}
$$

Using (22) we obtain initial conditions for functions $P^{ \pm}$ and $S^{ \pm}$:

$$
P^{ \pm}\left(0, y^{ \pm}\right)=p_{0}^{ \pm}\left(y^{ \pm}\right), S^{ \pm}\left(0, z^{ \pm}\right)=s_{0}^{ \pm}\left(z^{ \pm}\right) \text {. }
$$

Let us assume that functions $p_{0}^{ \pm}, s_{0}^{ \pm}$are periodic with the period $2 \pi$ and have continuous derivatives

$$
p_{0}^{ \pm}(x), s_{0}^{ \pm}(x) \in C_{2 \pi}^{1}(R) .
$$

For brevity of the presentation without a loss of generality, we can assume that

$$
\int_{0}^{2 \pi} p_{0}^{ \pm}(x) d x=0, \int_{0}^{2 \pi} s_{0}^{ \pm}(x) d x=0 .
$$

Theorem. There exist positive constants $\tau_{0}, \varepsilon_{0}$ such as that

1) Problems (2), (4), (24) have a unique solution for $(t, x, \varepsilon) \in\left[0, \frac{\tau_{0}}{\varepsilon}\right] \times R \times\left[0, \varepsilon_{0}\right]$;

2) Problems (23), (27) have a unique solution for $\left(\tau, y^{+}, y^{-}, z^{+}, z^{-}\right) \in\left[0, \tau_{0}\right] \times R^{4}$

3) For any $\eta>0$ there exists $\varepsilon_{\eta}>0$ such as that for all $\varepsilon \in\left(0, \varepsilon_{\eta}\right\rfloor, t \in\left[0, \frac{\tau_{0}}{\varepsilon}\right], x \in R$ the following estimates are satisfied

$$
\begin{gathered}
\left|u_{t}-\frac{1}{2}\left(p^{+}+p^{-}\right)\right|<\eta, \mid u_{x}-\frac{1}{2 c_{p}}\left(p^{+}-p^{-}\right)<\eta, \\
\left|v_{t}-\frac{1}{2}\left(s^{+}+s^{-}\right)\right|<\eta,\left|v_{x}-\frac{1}{2 c_{s}}\left(s^{+}-s^{-}\right)\right|<\eta .
\end{gathered}
$$

\section{Analysis of the resonance effect}

The averaged system (23) gives us a posibility to find conditions when the non-linear resonant interaction can take place.

\subsection{Non-resonance case}

Let us assume that for any $k, l \in Z$ the following condition is satisfied

$$
\frac{c_{p}}{c_{s}} \neq \frac{k}{l},
$$

ie $\begin{gathered}c_{p} \\ c_{s}\end{gathered}$ is an irrational number. Then the right-hand side of system (23) is equal to zero and the system can be reduced to four decoupled equations of the Burger type

$$
\begin{aligned}
& P_{\tau}^{ \pm}-\frac{a_{1}}{4 c_{p}^{2}} P^{ \pm} P_{y^{ \pm}}^{ \pm}=0, \\
& S_{\tau}^{ \pm}-\frac{b_{3}}{4 c_{s}^{2}} S^{ \pm} S_{z^{ \pm}}^{ \pm}=0 .
\end{aligned}
$$

The solution of Burger's equation is uniformly bounded but for a large $\tau$ it may not have a unique solution and a shock wave can be formed. Hence our analysis gives a region of validity of the non-linear model (12).

\subsection{Resonance case}

If we have that

$$
\frac{c_{p}}{c_{s}}=\frac{k}{l},
$$

then there is a possibility of resonant interaction between the waves. Let us consider the problem (12) with

$$
\rho=\mu=1, \lambda=2, a_{i}=b_{i}=1, i=1,2,3 \text {. }
$$


Then we obtain that $c_{p}=2$ and $c_{s}=1$. The averaging is done in the interval $[0,2 \pi]$ and we get the system of equations

$$
\left\{\begin{array}{l}
P_{\tau}^{+}-\frac{1}{2} P^{+} P_{y^{+}}^{+}=\frac{1}{\pi} \int_{0}^{2 \pi} \frac{\partial}{\partial y^{+}}\left(S^{+}\left(\tau, y^{+}-r\right) S^{-}\left(\tau, y^{+}-3 r\right)\right) d r \\
-\frac{1}{4 \pi} \int_{0}^{2 \pi} \frac{\partial}{\partial y^{+}}\left(P^{-}\left(\tau, y^{+}-2 r\right)\left(S^{+}\left(\tau, y^{+}-r\right)-S^{-}\left(\tau, y^{+}-3 r\right)\right) d r,\right. \\
P_{\tau}^{-}-\frac{1}{2} P^{-} P_{y^{-}}^{-}=\frac{1}{\pi} \int_{0}^{2 \pi} \frac{\partial}{\partial y^{-}}\left(S^{+}\left(\tau, y^{-}+3 r\right) S^{-}\left(\tau, y^{-}+r\right)\right) d r \\
+\frac{1}{4 \pi} \int_{0}^{2 \pi} \frac{\partial}{\partial y^{-}}\left(P^{+}\left(\tau, y^{-}+2 r\right)\left(S^{+}\left(\tau, y^{-}+3 r\right)-S^{-}\left(\tau, y^{-}+r\right)\right)\right) d r, \\
S_{\tau}^{+}-S^{+} S_{z^{+}}^{+}=\frac{1}{4 \pi} \int_{0}^{2 \pi} \frac{\partial}{\partial z^{+}}\left(P^{+}\left(\tau, z^{+}+r\right) P^{-}\left(\tau, z^{+}-r\right)\right) d r \\
-\frac{1}{4 \pi} \int_{0}^{2 \pi} \frac{\partial}{\partial z^{+}}\left(S^{-}\left(\tau, z^{+}-2 r\right)\left(P^{+}\left(\tau, z^{+}+r\right)-P^{-}\left(\tau, z^{+}-r\right)\right)\right) d r, \\
S_{\tau}^{-}-S^{-} S_{z^{-}}^{-}=\frac{1}{4 \pi} \int_{0}^{2 \pi} \frac{\partial}{\partial z^{-}}\left(P^{+}\left(\tau, z^{-}+3 r\right) P^{-}\left(\tau, z^{-}-r\right)\right) d r \\
-\frac{1}{4 \pi} \int_{0}^{2 \pi} \frac{\partial}{\partial z^{+}}\left(S^{-}\left(\tau, z^{+}-2 r\right)\left(P^{+}\left(\tau, z^{+}+r\right)-P^{-}\left(\tau, z^{+}-r\right)\right)\right) d r .
\end{array}\right.
$$

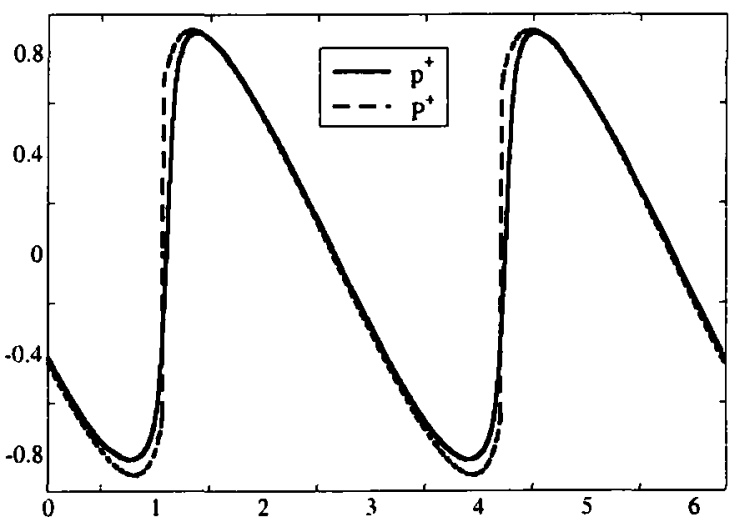

Fig 2. The solution $p^{+}$and the asymptotic $P^{+}$

\section{Conclusions}

In this paper the analysis of limitations of the linear elastic waves model is done and a new method for modelling the interaction of non-linear waves is proposed. The comparison with linear models gives the following conclusions.

1. The linear-wave approximation is accurate only till time moments $t \ll \varepsilon^{-1}$, where $\varepsilon$ is the wave amplitude.

It is important to note that this system must be solved only once and the obtained solution can be used to approximate the solution of problem (12) for any $\varepsilon$, by using appropriate independent variables

$$
\tau=\varepsilon, y^{ \pm}=x \pm c_{p} t, z^{ \pm}=x \pm c_{s} t .
$$

Numerical algorithms for Burger's equation are well-developed and the system is solved in a finite domain $\left[0, \tau_{0}\right] \times[0,2 \pi]$.

The possibility of developing resonant waves in the system describing elastic waves was also investigated in [16]. Here the analysis is done by using the regular expansion scheme, which iteratively takes into account the effects of small non-linear disturbances. It leads to the solution expansion given in the form (16). We have proved above that such an approximation is uniformly valid only for $\varepsilon t \ll 1$. The resonance analysis given in [18] leads to the same condition (30). It is obvious that the regular expansion method cannot be used to approximate the solution for $\varepsilon t=O(1)$.

Fig 2 shows the solution $p^{+}$of system (12) and the asymptotic approximation $P^{+}$for $t=\frac{1}{\varepsilon}, \varepsilon=0,01$. We see that the averaged system (23) approximates uniformly the initial system till time moments $t=O\left(\frac{1}{\varepsilon}\right)$ and the effect of resonant interaction is also identified correctly.

2. If $t \approx \varepsilon^{-1}$, then the non-linear effects become important. Mathematical modelling of this phenomena requires solving complicated systems of non-linear PDE.

3. If $\frac{c_{p}}{c_{s}}$ is an irrational number, then the resonant interaction between waves is not taking place and oscillations of the solution are described by independent non-linear travelling waves and the amplitudes of these waves are changing slowly.

4. If $\begin{aligned} & c_{p} \\ & c_{s}\end{aligned}$ is a rational number, then the system describes the non-linear interaction of waves and the resonance phenomena take place. In this case we need to solve a system of four integrodifferential equations. Though the description of this new problem is more complicated than the initial one, but the numerical analysis of this problem is even easier (eg $[19,20])$. In addition we note, that the problem (23), (27) must be solved only once and the obtained solution can be used for any value of the parameter $\varepsilon$. We note, that in this paper our method is applied for a very particular elastic problem. Therefore it a would be important to apply it for more general elastic problems. 


\section{References}

1. Samarskii A. A.; Mikailov A. P. Mathematical Modelling: Ideas. Methods. Examples (Математическое моделирование: Идеи. Методы. Примеры). Moscow: Fizmatlit, 2001. 302 p. (in Russian).

2. Johnson R. S. A modern introduction to the mathematical theory of water waves. Cambridge: Cambridge University Press, 1997. 461 p.

3. Whitham G. B. Linear and nonlinear waves. New York: John Willey \& Sons Inc., 1999. 656 p.

4. Bedford A.; Drumheller D. S. Introduction to elastic wave propagation. New York: John Willey \& Sons Inc., 1996. $312 \mathrm{p}$.

5. Kulikovskii A. and Sveshnikova E. Nonlinear waves in elastic media. Florida: CRC Press Inc., Boca Raton, 1995. $256 \mathrm{p}$.

6. Antman S. S. Nonlinear problems of elasticity. Appl. Math. Sci., Vol 107. Berlin and New York: Springer - Verlag, 1995. xviii+750 p.

7. Engelbrecht J.; Nigul U. Nonlinear deformation waves (Нелинейные волны деформации). Moscow: Nauka, 1981. 256 p. (in Russian).

8. Anile A.M., Hunter J.K., Pantano .P and Russo G. Ray methods for nonlinear waves in fluids and plasmas. Harlow, England: Longman scientific and technical, 1993. 243 p.

9. Nayfeh A. H. Perturbation methods. New York: John Willey \& Sons Inc., 2000. $448 \mathrm{p}$.

10. Nayfeh A. H. The general problem of elastic wave propagation in multilayered anisotropic media. J. Acous. Soc. Am., Vol 89(4), 1991, p. 1521-1531.

11. Hunter J. K. Interaction of elastic waves. Stud. Appl. Math., Vol 86, 1992, p. 281-314.
12. Embid P. F. and Majda A. Averaging over fast gravity waves for geophysical flow with arbitrary potentional vorticity. Comm. P. D. E., Vol 21, 1996, p. 619-658.

13. Yong D. H. and Keverkian. Solving boundary-value problems for systems of hyperbolic conservation laws with rapidly varying coefficients. Stud. Apll. Math., Vol 108, 2002, p. 259-303.

14. McCall K. R. Theoretical study of nonlinear elastic wave propagation. J. Geophys. Res., Vol 99, 1994, p. 2591-2600.

15. Koen E-A; Van Den Abeele K. Elastic pulsed wave propagation in media with second or higher order nonlinearity. Part I. Theoretical framework. J. Acoust. Soc. Am., Vol 99, 1996, p. 3334-3345.

16. Landau L. D.; Lifshitz E. M. Theory of elasticity: course of theoretical physics, Oxford: Butterworth - Heinemann, 1986. $187 \mathrm{p}$.

17. Krylovas A. and Ciegis R. Asymptotic approximation of hyperbolic weakly nonlinear systems, Journal of Nonlinear Mathematical Physics, Vol 8, No 4, 2001, p. $458-470$.

18. Krylovas A. and Čiegis R. Asymptotical analysis of one dimensional gas dynamics equations. Mathematical Modelling and Analysis, Vol 6, No 1, 2001, p. 103-112.

19. LeVeque R. J. Numerical methods for conservation laws (Lectures in Mathematics). Basel: Birkhauser Verlag, 1992. $214 \mathrm{p}$.

20. LeVeque R. J. CLAWPACK user notes. Department of Applied Mathematics, University of Washington, 1997. Software available from the web at http:// www.amath.washington.edu/ rjl/clawpack.html. 